\title{
Traditional Thai Music Competition: The Culture of Pipat Competition
}

\section{Veera Phansue}

Department of Music Education, Faculty of Fine Arts, Srinakharinwirot University, Bangkok, Thailand. Email : veerap@swu.ac.th

\begin{abstract}
The title of this research is "The Culture of Pipat Competition" has three purposes as follows: 1) To study the knowledge of Pipat competition; 2) To study the process of Pipat competition; 3) To study the process of judging and deciding a Pipat competition.

This research used the qualitative method and presented the research by using descriptive analysis. The results found that; 1) The knowledge of Pipat competition. The competition is to bring ensembles to perform their skills and abilities. Mostly, in a Pipat ensembles the knowledge and skills displayed are governed by a definite set of form and rules during the competition. Only the Pipat Mon that there is no any form or rule in the competition. The ensembles that want to join the competition have to prepare very well in term of musicians and place for rehearsal before the event. Moreover, the conductor of ensemble has to be highly skilled and experienced in coming up with a new technique for playing in order to take an advantage over the rivals. And the conductor should know the strength and weakness of the rivals too.
\end{abstract}

2) The Study of the process of Pipat competition. The competition has a certain set of forms and rules. The detail of forms and rules will be set by each competitor agreement and it is very easy for practice. It is different from the competition that there is no form or rule so, this kind of competition, the musicians should have a lot of knowledge and in an easy and a relax ways without strict rule, form or time in the competition many kinds of songs will be played. Therefore the conductor must plan everything for the competition. There are 2 types of competition; 1 ) different song, each ensembles will take turn to play a song and begin with the basic melody until the highly complicated notes and those songs must be in the same style. 2) same song, each ensemble plays same song simultaneously during the whole competition. This kind of competition is very serious and if any of the ensemble cannot play or keep pace with the melody it means they lose the competition.

3) Study of the process of deciding and judging a Pipat competition. There are 3 types of judging in the Pipat competition.

1. The musician and the singer know the result by themselves.

2. The audience decides who wins the competition.

3. The judge decides who wins the competition.

The advantage of decision is choosing the best ensemble with developed technique and playing method while the disadvantage is when the musicians do not accept the decision often leading to quarrel and disharmony.

Keywords : Pipat ensemble, Pipat Competition, Culture of Traditional Thai Music Competition. 


\section{INTRODUCTION}

Thai Pipat ensemble is an ensemble which has developed in term of the instruments and forms since Sukhothai to Rattanakosin era. The Pipat ensemble has had a duty to serve all levels of society. Traditional Thai Music became very successful, especially during the reign of Rama V until prior the revolution in 1932 which is considered the golden age of music in Thailand. In those days, if there were occasions such as a Shaving ceremony and funeral, several ensembles would be hired to play. Each band would play up to the payer's orders. Ensembles also had the opportunity to compete in events. The Pipat ensemble competition was known among the musicians and local people. The competitions are common for solo instruments or playing ability. The winners also bring acclaim to their affiliations.

In the past, traditional Thai music bands were under the patronage of the royal courts or other houses of learning. Meanwhile, each house would find famous skilled musicians to teach the band in its own house. Anyway, talented Thai musicians were plentiful in Bangkok and in other provinces as well. So there was a good chance for unknown musicians but very skillful to performed their abilities. The stage for competition was not hard to find as they happened at various places. The competitions at that time, had a lot of audiences who generally included the royal family and the general public. The winning Pipat ensemble became famous all over the place. The traditional Thai Music band competitions flourished and peaked at this time.

Competitions are to decide who is better or more powerful than the others. To compete, competitors need finesse and innovation in the competitions with a lot of rehearsal and good teaching for helping to develop those who struggle to gain high confidence. Therefore, the Pipat ensemble competition is a process to develop musical skills for improving music ability, brand adjustment, and harmony. It is believed that the Pipat ensemble competition is one of the most important processes that contributes to the creation of Traditional Thai Music bands.

Currently, the format of the Pipat ensemble competition has changed from the past. The characters of the competitions are different depending on the social context and their popularity. Plus other cultures move into Thai society and that makes various Thai traditional entertainments include the Pipat ensemble competitions are not as popular as before. Therefore, this research is to study the culture of the Pipat ensemble competitions from past to present.

\section{The Aim of the Research is as follows:}

1. To study Pipat ensemble competitions.

2. To study the processes of Pipat ensemble competitions.

3. To study the judgments in Pipat ensemble competitions.

\section{MATERIALS AND METHODS}

The researcher divided the process of action research as follows: 


\section{The collection of information.}

To study Pipat ensemble competitions, researcher studied documents and related researches from libraries and educational institutions, government and regulatory agencies such as;

- Central Library, Srinakharinwirot University.

- Research Institute for Languages and Cultures for Rural Development Library, Mahidol University.

- H.R.H. Princess Maha Chakri Sirindhorn Music Library. Etc.

\section{Collecting field data.}

2.1 Preparation for field work.

- Researcher prepared by writing a data plan to cover all the contents which are related to the competitions.

- Researcher prepared field devices such as video recorders, cameras, audio recorders, and Mini disks in order that they are in a state ready for use.

2.2 Selection the study area.

- Researchers have defined the study area as Bangkok, Thailand with a group of musicians who have experience in the competitions.

\section{The data analysis.}

Data from the field studies to analyze are as follows;

3.1 Knowledge of the Pipat ensemble competitions.

- To get a job in the Pipat ensemble competitions.

- How Pipat musicians position themselves in an ensemble.

- How a Pipat ensemble practices for a competition.

- How to study a competitor's band.

- How to prepare the songs to use in the competitions.

3.2 The processes of Pipat ensemble competitions.

- The strategies used in the Pipat ensemble competitions.

- The flexibility for a band to play first as "Wong-Tang" or second as "Wong Par Chan" in competitions.

3.3 The judgments in Pipat ensemble competitions

- The results of the competitions.

- The advantages and disadvantages of the competitions.

\section{Summary and discussion.}

4.1 Conclusion to the findings from the culture of the Pipat ensemble competitions.

\section{DISCUSSION}

\section{To study Pipat ensemble competitions.}

1.1 Knowledge of Pipat ensemble competitions.

The Pipat ensemble competition means having two bands play alternately. The band which plays first is called "Wong Tang" the band which plays after is called "Wong Par Chan". Musicians who play in the competitions need to have excellent and 
advanced music skills with experience in Thai musical conventions. Good competitors with similar skills will make the competitions more enjoyable. After the competitions, musicians will study the latest performance to learn their mistakes and find new strategies and techniques to use for the next competition.

Pipat ensemble Competitions in the past and in the present are divided into two types. The first is impromptu competitions which the competitors have no time to prepare. The second type is announced in advance that there will be a competition so the competitors can prepare ahead.

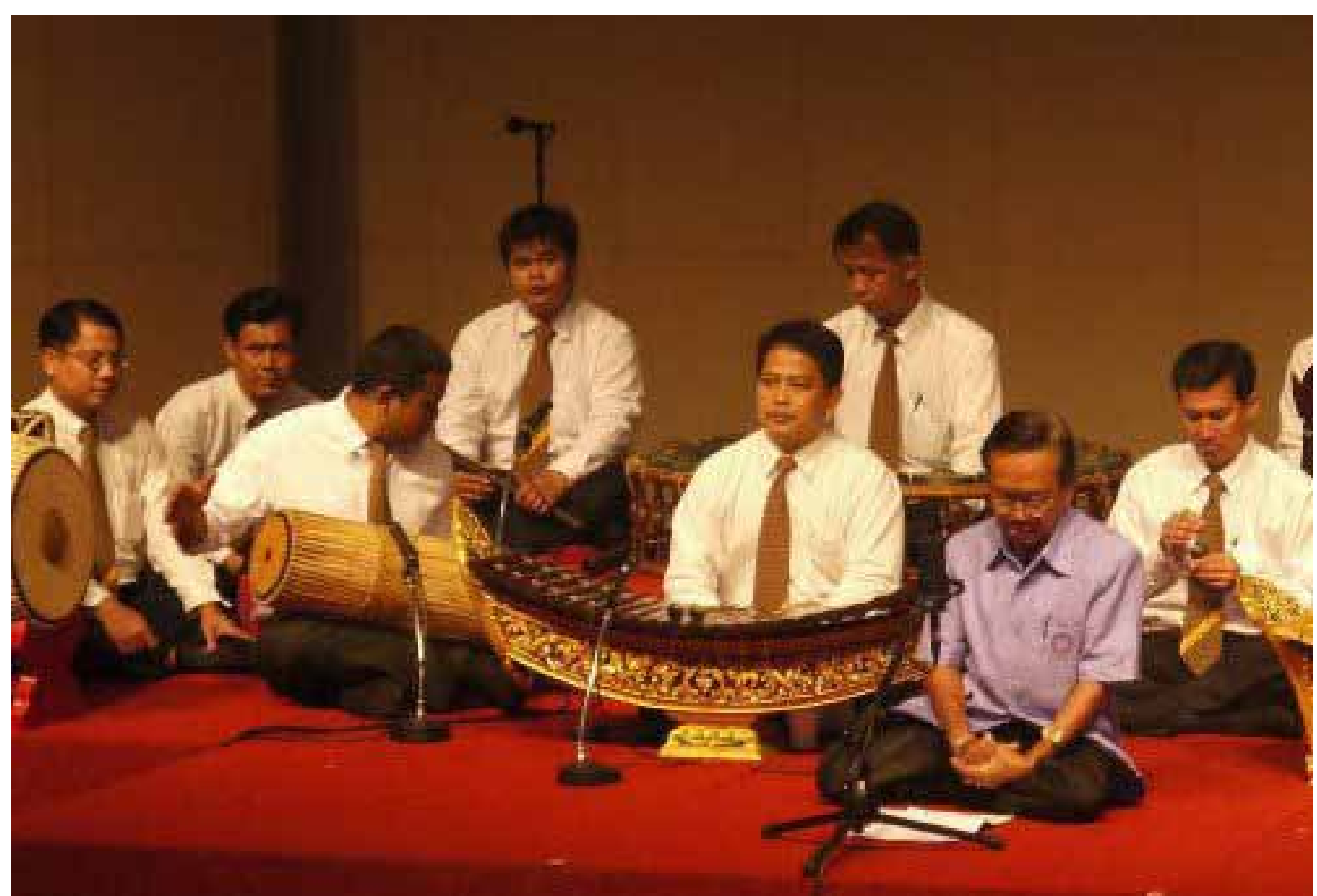

Figure 1. Thai Traditional Music: Pipat Ensemble

1.2 To get a job in the Pipat ensemble competitions.

To get a job in the Pipat ensemble competitions, the students or musicians in the band should come from the same peer or used to practice together, and have advanced skills in music and then they can be considered. There are a variety of tasks and activities in the Pipat ensemble competitions such as;

- Activities that the host wants to have the Pipat ensemble competition such as, funerals, annual matriculation, or paying respect to the goddess "Wai Kru" ceremony etc.

- Demonstrations of the Pipat ensemble competition.

- Activities or projects for the Pipat ensemble and competitions by education agencies for various occasions.

- Different kinds of Pipat ensembles, such as; Pipat Sapa ensemble, Pipat Mon ensemble or Pipat Nang Hong ensemble.

- The duration of competitions.

- The number of songs used in the competitions. 
Getting ready for competitions means the readiness of the players and singers through the overall look of the entire band.

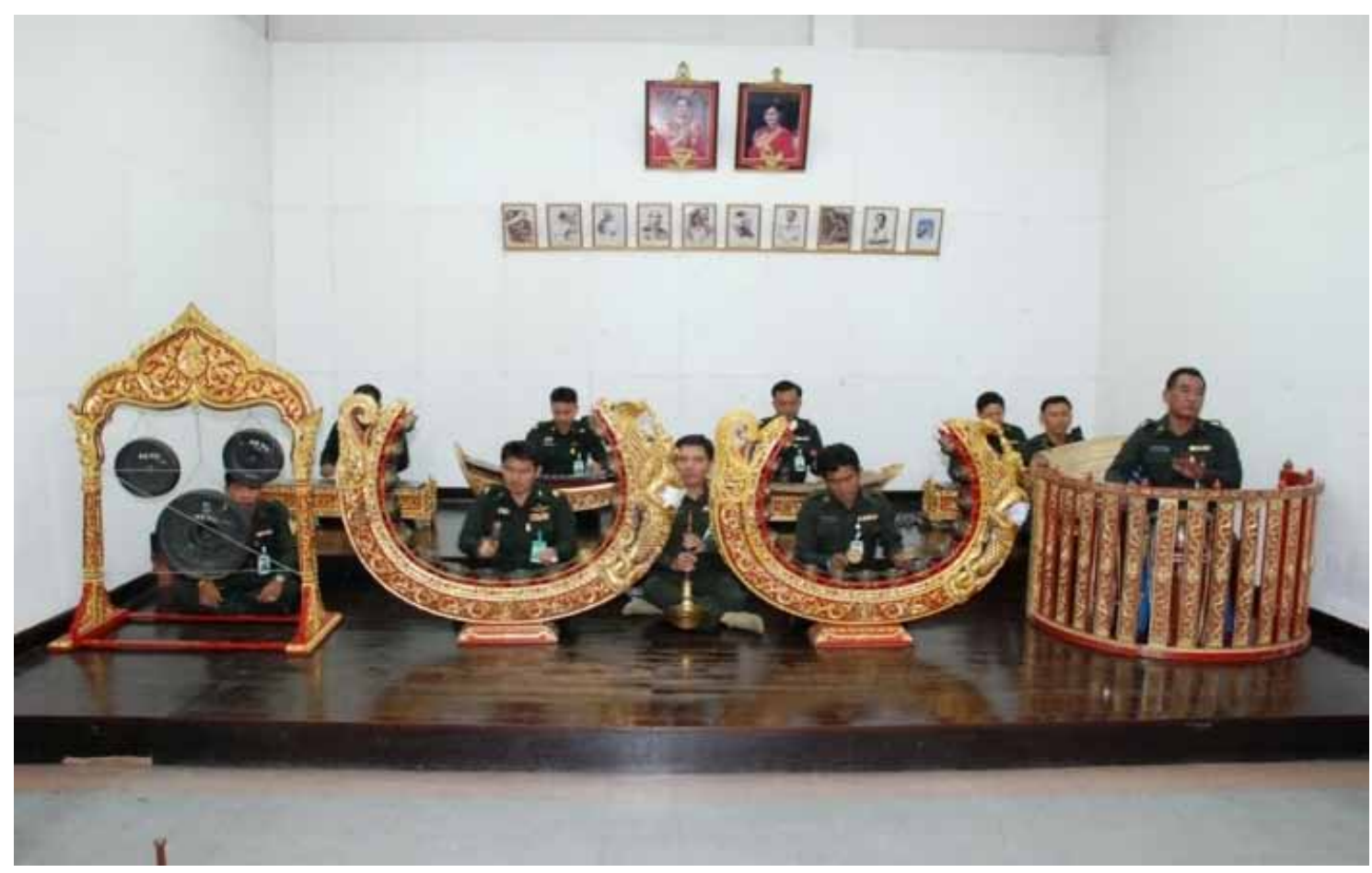

Figure 2. Pipat Mon: one kind of Thai Traditional Thai Music Ensemble

1.3 The positions of the musicians in the Pipat ensemble.

The duties and position of the musicians in a Pipat ensemble are given by the head of the Pipat ensemble whose job is to control and improve. The head of the Pipat ensemble will also know the potential of the musicians in the band. It is important thing to consider the alignment or position of the musicians or singers follow the agreement or the rules of competitions. Therefore, the head of the band will consider and arrange all musicians, instruments, duties and positions. Every band will have the form that contains of similar duties and position as these follows:

- The singers need to have a good voice, bright, clear tone, and resonant vocals for both women and men.

- The alto-xylophone (Ranat-Ek) is a leader of the band and whoever plays the alto-xylophone in the competitions should have a higher skill than other band members.

- The musician who plays the cymbal (Ching) need to know the song and keep the rhythm steady and lack of mistake. So there must be quite experience and significance in this position. It may say Ching is the second importance to the alto-xylophone.

- The leather drums, if a barrel drum (Klong Song-Na), hit with single strikes without noise interference so not to change the speed of the song. However, if using a double-headed barrel drum (Klong Khaek) the drummer must not hit too quickly so that the music does not get out of control and end the competition. The roles of the leather drums need to be clearly understood, in that the leather drums in the band is only used for rhythm. 
- Lower gong circle (Khong Wong Yai) has to accurately recognize the music as it plays the melody for the other instruments.

- Higher gong circle (Khong Wong Rek) must have a clear melody.

- Lower xylophone (Ranad Thum) also has style and good melody to impress the audience.

- The speed at which each piece of music is played changes with each type of song.

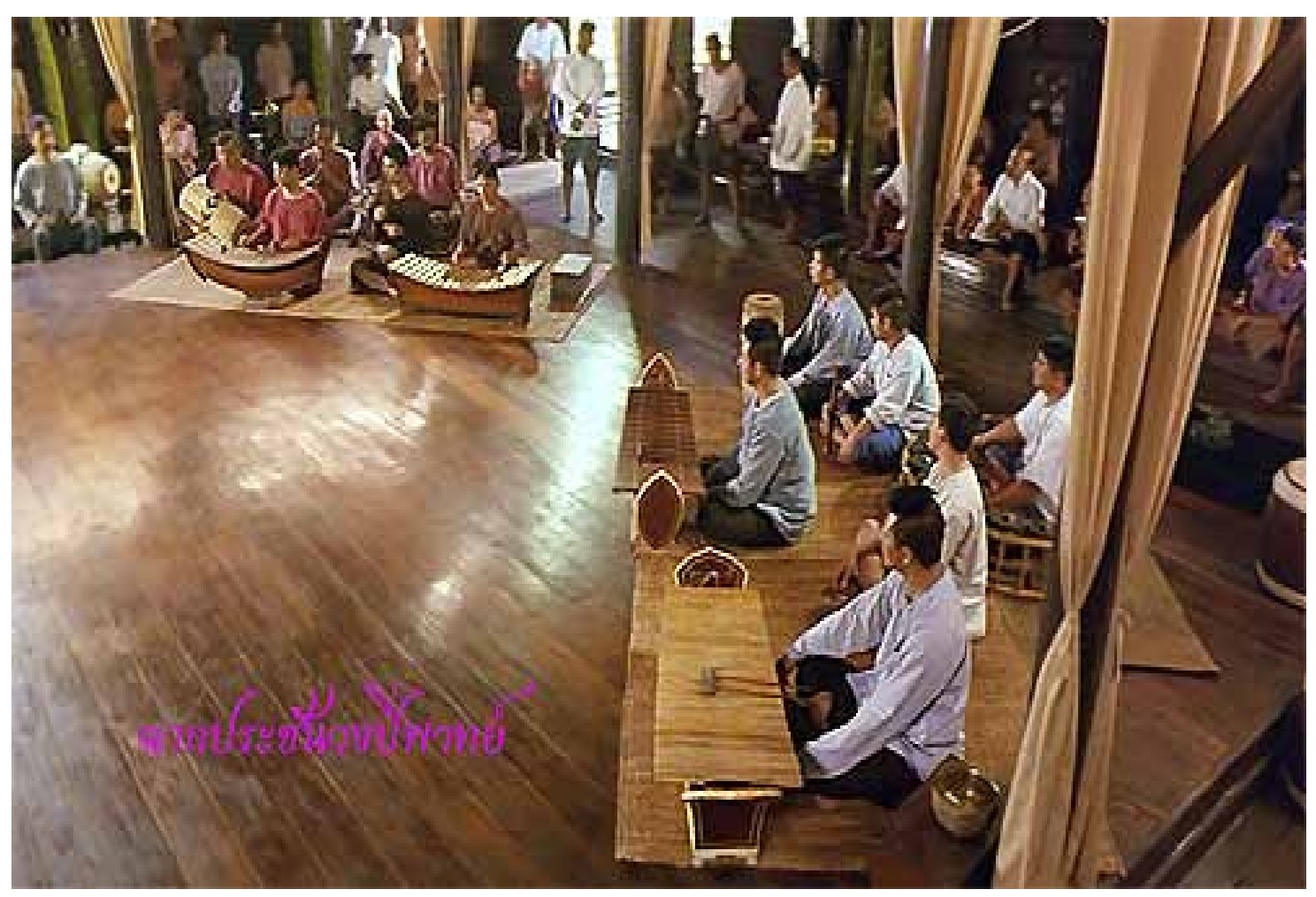

Figure 3. Pipat Competition

For the competitions, the competitors must be ready and have unity as a group. If each instrument has a skilled musician, it will give an advantage to the competitor.

1.4 The resources to practice for the competitions of the Pipat ensemble.

Practicing is an important part of music. In order to play the music, they need to practice beforehand. Practicing makes the player and the singer understand the details of the song such as the instrumental interlude or how to use speed to play. In addition, the head of the Pipat ensemble will have to adjust to the rules that have been set up. Every musician in the Pipat ensemble will have three qualities, which are;

- Responsibility

- Discipline

- Punctuality

Duties of the Pipat ensemble and the head of Pipat ensemble are as follows:

- Telling everyone who will enter the competitions to understand that they need to practice separately before practicing together as a band.Each player takes their own responsibility to practice for the first term.

- Define and calculate the proper time to practice together. 
- To practice together as a band, the head of the band needs time to improve and correct the musicians. In addition, they need to check the lyrics and the singers including the instrumental interlude of Pipat ensemble and make sure various details are clear.

- Do not change the structure or the sentence of the songs that the composer meant. It is a faux pas and extremely rude to do that. The band is better find an expert to double check the music and make sure everything is properly completed.

- Readiness is essential. The band must have trained and practiced as much as possible.

1.5 To study the rival ensemble.

In competition, the head of the Pipat ensemble should deeply and carefully study the rival. Particular their approaches and strategies of playing the alto-xylophone (Ranat-Ek) which are the keys to success the competition. This rival estimation should include the ability of each instrument of the competitors in the whole competition. The assessment is as follows;

- Consider the readiness of the musicians in the competing ensemble.

- Consider the sounds of the instruments in the competing ensemble.

- Which melody that the rival uses in the songs.

1.6 Preparation the songs for using in the competitions.

The regulations state that for the Pipat Sapa ensemble competition, the band shall start by playing the overture called "Hom-Rong-Sapa", Pa-ma-Ha-Ton song, Ja-Ra-KeaHang-Yao song, Sri-Boud song, Bu-Ran song, Ta-yoi song, and then each instrument plays a solo and finally the last song called "Pleng-Ra". In a temple fair the band will use the Pipat Mon ensemble to play in competition. The head of the Pipat Mon ensemble need to prepare a Mon song such as Tao songs (Pleng-Tao) and Ta-Yoi songs (Pleng-Ta-Yoi). The musician will practice a solo from basic songs to more complex songs such as Glao-Nai song (Pleng-Glao-Nai) and Ta-Yoi-Diao song (Pleng-Ta-YoiDiao). However, in competition the bands will play well-known and popular songs or Hang-khrueang songs (Pleng-Hang- khrueang) at the end of competition to make it a more enjoyable.

Preparing the song for a competition is as follows;

1. The precision of the songs while playing in the competitions.

2. Readiness of the band.

3. Formalize.

4. The structure of the songs.

5. The way to use Hang- khrueang songs (Pleng Hang- khrueang) compared with Tao songs (Pleng-Tao).

\section{A study of the processes of Pipat ensemble competitions.}

2.1. The strategy used in Pipat ensemble competitions.

There are two parts in the strategy which are used in Pipat ensemble competitions:

1. Playing and singing in practice and the adjustment of the band. 
2. To solve immediate problems.

The strategy in Pipat ensemble competitions starts by playing a Prob-Kai song (PlengProb-Kai) such as Pa-ma-Ha-Ton song, Ja-Ra-Kea-Hang-Yao song, Sri-Boud song, or Bu-Ran song. Then, a Ta-yoi song such as Kaat-Lob-Bu-Ri song, Kha-Man-Rad-Bu-Ri song, Ta-Yoi-Yuan song, Ta-Yoi-Kha-Man song, Ta-Yoi-Nok song, Ta-Yoi-Nai song is played. After that, a solo from each instrument is played.

The general strategies are as follows:

1. Start to play basic songs to more complex song.

2. Use the same Hang- khrueang songs (Pleng-Hang- khrueang) as they will use in competition.

3. Play a solo from each instrument with medium rhythms in Ta-Yoi songs (Pleng-Ta-Yoi) only. If playing a Glao-Nai song (Pleng-Glao-Nai) and a Ta-Yoi-Diao song (Pleng-Ta-Yoi-Diao) then a Hang- khrueang songs (PlengHang- khrueang) must only be played at the end.

2.2. The process of flexibility for a band to play first as "Wong-Tang" or second as "Wong Par Chan" in competitions.

The position of a band playing first as "Wong-Tang" or second as "Wong-Par-Chan" in the Pipat Sa-Pa ensemble competitions depends on the agreement, such as using a raffle to decide which band plays first etc.

When the band is "Wong-Tang" it means the band plays first. According to ancient tradition, Wong-Tang must play an overture called "Hom-Rong-Sapa" first.

When the band is "Wong-Par-Chan" it means the band plays second and is called "Wong-Par-Chang". The band needs to handle with any song in every situation without panicking or rushing.

1. If the band is "Wong Tang", they should not use too complex song for testing the rival.

2. When playing a solo song, there must be a solo from each instrument within the same song after the Tao song (Pleng Tao).

3. Do not use a song or melody that the competitor band has a lot of experience.

The flexibility for a band to play first "Wong-Tang" or to play second "Wong-ParChan" in Pipat Sa-Pa ensemble competitions depends on the ability of the musicians in the band. The band uses the solo songs and Hang-khrueang songs (Pleng-Hangkhrueang) as the songs in the competition.

1. If the band is "Wong Tang", the musicians need to find a highly technical piece of music because they play first.

2. If the band is "Wong Par Chan", the musicians need to find a higher level song to play in the competition.

To compete in the band competition:

1. Play the same song and improvise the melody.

2. Play many different songs and know the songs to compete.

Sometimes the bands use the song names which have similar meaning in the competition. For example, the song names have similar actions or consonance sound 
or they use the name of the song that begins with connecting words such as bird or serpent deity that would be a song about nature. Using the meaning of the name of a song to compete is a priority, the band may use A-Noo song which means child (Pleng A-Noo) compete with A-Hear song which means brother (Pleng A-Hear), A-Hear song (Pleng A-Hear) compete with Peh song which means uncle (Pleng Peh) and Peh song (Pleng Peh) compete with Chin-Sair song which means doctor or teacher (Pleng ChinSair) etc.

In competitions the Pipat Sa-pa ensemble will compete by using lyrics of songs from the same literature. The instruments play the same melody as the lyrics or compose new lyrics in the same song in the competition etc.

In competitions the Pipat Sa-pa ensemble will compete by using the same sound or dialect of song. For example, if Wong Tang uses a Chinese sound to compete, Wong Par Chan have got to use a Chinese song to compete in the competition too etc.

In competitions the Pipat Sa-pa ensemble will compete by using the same drum patterns (the patterns to hit a drum) called "Na-Tab". For example, if Wong Tang uses the song in "Na-Tab-Mon" (the drum uses a Mon pattern to play the song) then Wong Par Chan must use the song in "Na-Tab-Mon" to compete in the competition etc.

\section{The Process of the Judgments in Pipat Ensemble Competitions.}

In the past, in a Pipat Sepha ensemble competition, the winner would not be announced. The musicians were expected to know who won the competition by themselves. After finishing the competition, the musicians greeted and accepted each other. There are 3 ways to decide the competition;

1. The musicians and the singers know the result by themselves.

2. The audience decides who wins the competition.

3. The judge decides who wins the competition.

\section{CONCLUSION}

The advantage of a decision is that the musicians can see the high standard and develop their technique. However, when the musicians do not accept the decision they may constantly quarrel with the other musicians which brings disunity and is a disadvantage of competition.

1. The culture of Pipat ensemble competition is an advanced music art of Traditional Thai Music

Each Pipat ensemble competition should be studied in various ways to benefit music education as follows:

- Finding a new technique from Pipat ensemble competitions.

- A view of society between musicians and audiences.

- The culture of the Pipat ensemble competition.

2. This research about the culture of the Pipat ensemble competition is from a musical dimension. It reflects the confidence in the culture of music and should be used to benefit the agencies or organizations, such as; 
- Libraries

- Office of Arts and Culture.

\section{REFERENCES:}

Atmadyakul, Poonpit. (1984). Traditional Thai Music Appreciation. Bangkok: Kiet Thurakit Press.

Boonmanum, Chalermsak. (1985). Theory of Traditional Thai Music.

Chalernchai, Ruang. (1986). Program Note of Thai Traditional Music Competition for the National Culture Supporting. Bangkok: Gurusapha Press.

Dharmavihara, Sangopsuk. (1997). Traditional Thai Music. The Press of Chulalongkorn. Inthanin, Pradit. (1998). Thai Classical Music and Dance. Bangkok: Suwiriyasan Press.

Jaem-arun, Niran. (2001). A Study of Traditional Thai Xylophone Music Solo in the Version of Master Boonyong Ketkong. Graduate Thesis in Ethnomusicology Program, Srinakharinwirot University: Bangkok.

Kaewsrl, Songwit. (1980). Traditional Thai Music: Structural, Technical Term, and Content Summary. Bangkok: Rungsilp Press.

Lenasawadhi, Apitchat. (2000). Traditional Thai Music String in Basic Practice. Bangkok: Sophon Press.

Office of the Higher Education Commission. (1995). Disciplines and professional Standard Criteria for Thai Musicians. Bangkok: Prakaipruk.

Pikunsri, Chalermsak . (1999). Theory of Traditional Thai Music. Bangkok: Odeon Store.

Phukan, Chanit. (2001). Traditional Thai Music Essence. Bangkok: Krungthon Patthana Press.

Phukhaothong, Sangnat. (1996). The Thai Traditional Music and Access to Thai Traditional Music. Bangkok: Reunkeaw Press.

Rungruang, Punya. (1978). History of Traditional Thai Music. Bangkok: Thai Watana Panich Press.

Samrongthong, Butsakorn. (1996). Variations of Traditional Thai Percussion Melodic Music Instruments. Bangkok: Chulalongkorn Press.

Sinlabapbaleng, Chin and Chindawattana, Likhit. (1978). Thai Music Education. Bangkok: Aksornchareontasana.

The Foundation of Luang Praditpairoh (Sorn Silabatbaleng). (1981). Thai Traditional Music Homage Ritual and Introduction of Traditional Thai Music. Bangkok: -.

Royal Institute of Thailand. (1997). Thai Traditional Music Term Encyclopedia in the Kita Sector. Bangkok: Maha Chula Press.

Tramote, Montri. (1971). Traditional Music. Bangkok: Department of Religious Press. Yoobhodhi, Thanit. (1987). Traditional Thai Music Instruments. Bangkok: Ganesha Press. . (1971). Somsongseang: Life of Thai Traditional Music. Bangkok: Department of Fine Arts Press. 